so that $|g(z)|=\left|\Gamma\left(\frac{1}{2}(x+7)+\frac{1}{2} i y\right)\right| \leq \Gamma\left[\frac{1}{2}(x+7)\right]$. By the concavity of the $\Gamma$ function, $|g(z)| \leq \max \left\{\Gamma\left(\frac{1}{2}(a+7)\right), \Gamma\left(\frac{1}{2}(-a+7)\right)\right\}, z \in \mathcal{E}_{\rho}$. Thus we have from (38) and (30),

$$
|E|<\frac{1}{2}(\pi a b)^{\frac{1}{2}} \max \left\{\Gamma\left(\frac{1}{2}(a+7)\right), \Gamma\left(\frac{1}{2}(-a+7)\right)\right\} \sigma_{G_{7}(a)} .
$$

The selection $a=5.0$ yields

$$
\begin{aligned}
|E| \leq \frac{1}{2}(8.772)(120)\left(3.867 \times 10^{-15}\right) \doteq 2.04 & \times 10^{-12} . \\
& \text { P. DAVIS } \\
& \text { P. RABINOWITZ }
\end{aligned}
$$

NBS

${ }^{1}$ S. Bergman, Kernel Functions and Conformal Mapping, Math. Surveys No. 5, New York, 1950

2'P. DAvis, "Errors of numerical approximation for analytic functions," $J n$. of Rational Mech. and Analysis, v. 2, 1953, p. 303-313.

${ }^{3}$ C. Lanczos, Introduction, Tables of Chebyshev Polynomials. NBS Applied Math., Ser. 9, Washington, D. C., 1952.

${ }^{4}$ A. N. Lowan, Norman Davids \& Arthur Levinson, "Table of the zeros of the Legendre polynomials of order 1-16 and the weight coefficients for Gauss' mechanical quadrature formula," Am. Math. Soc., Bull., v. 48, 1942, p. 739-743. formula.

${ }^{5}$ See, e.g., W. Magnus \& F. Oberhetringer, Formeln und Sätze, 2nd ed., p. 3, 1st

\title{
Integrals Occurring in Problems of Molecular Structure
}

In theoretical work on molecular structure based either upon the valence bond method or upon the molecular orbital method, the electronic wave functions are usually built up from atomic orbitals (AO's). The calculation of most physical and chemical quantities then reduces to the evaluation of a number of integrals involving these orbitals. It is customary to adopt SLATER $^{1}$ type AO's, defined by (cf. RüDENBERG ${ }^{2}$ )

$$
\begin{aligned}
(1 s) & =\left(\zeta^{3} / \pi\right)^{\frac{1}{3}} e^{-\zeta r} & (3 d \bar{\pi}) & =\left(2 \zeta^{7} / 3 \pi\right)^{\frac{1}{3}} y z e^{-\zeta r} \\
(2 s) & =\left(\zeta^{5} / \pi\right)^{\frac{1}{2}} r e^{-\zeta r} & (3 s) & =\left(2 \zeta^{7} / 5 \pi\right)^{\frac{1}{3}}\left(r^{2} / 3\right)^{-\zeta r} \\
(2 p \sigma) & =\left(\zeta^{5} / \pi\right)^{\frac{1}{2}} z e^{-\zeta r} & (3 p \sigma) & =\left(2 \zeta^{7} / 15 \pi\right)^{\frac{1}{3}} z r e^{-\zeta r} \\
(2 p \pi) & =\left(\zeta^{5} / \pi\right)^{\frac{1}{2}} x e^{-\zeta r} & (3 p \pi) & =\left(2 \zeta^{7} / 15 \pi\right)^{\frac{1}{3}} x r e^{-\zeta r} \\
(2 p \bar{\pi}) & =\left(\zeta^{5} / \pi\right)^{\frac{1}{3}} y e^{-\zeta r} & (3 p \bar{\pi}) & =\left(2 \zeta^{7} / 15 \pi\right)^{\frac{1}{3}} y r e^{-\zeta r} \\
(3 d \sigma) & =\left(\zeta^{7} / 2 \pi\right)^{\frac{1}{3}}\left(z^{2}-r^{2} / 3\right) e^{-\zeta r} & (3 d \delta) & =\left(2 \zeta^{7} / 3 \pi\right)^{\frac{1}{3}} \frac{1}{2}\left(x^{2}-y^{2}\right) e^{-\zeta r} \\
(3 d \pi) & =\left(2 \zeta^{7} / 3 \pi\right)^{\frac{1}{3}} x z e^{-\zeta r} & (3 d \bar{\delta}) & =\left(2 \zeta^{7} / 3 \pi\right)^{\frac{1}{3}} x y e^{-\zeta r}
\end{aligned}
$$

where $(x, y, z)$ are the cartesian coordinates of the electron referred to the nucleus as origin with the $z$-axis directed along the internuclear axis towards the other nucleus, $r^{2}=x^{2}+y^{2}+z^{2}$ and $\zeta$ is a numerical screening parameter. Occasionally complex Slater type AO's are used; however since these are simply linear combinations of the real Slater AO's, no distinction will be made between integrals involving real and complex orbitals.

Of the distinct types of two-centre integrals that arise, six are of major 
importance; if $\chi_{a}, \chi_{a^{\prime}}$ etc. denote orbitals of nucleus $a$ and $\chi_{b}, \chi_{b^{\prime}}$ etc. orbitals of nucleus $b$, these are*

Overlap integrals:

$$
\left(\chi_{a} \mid \chi_{b}\right)=\int \chi_{a} \chi_{b} d v
$$

Direct Coulomb attraction integrals:

$$
\left(\chi_{a}\left|1 / r_{b}\right| \chi_{a}\right)=\int \chi_{a}\left(1 / r_{b}\right) \chi_{a} d v
$$

Exchange Coulomb attraction integrals:

$$
\left(\chi_{a}\left|1 / r_{b}\right| \chi_{b}\right)=\int \chi_{a}\left(1 / r_{b}\right) \chi_{b} d v .
$$

Coulomb repulsion integrals :

$$
\left(\chi_{a} \chi_{b} \mid \chi_{a^{\prime}} \chi_{b^{\prime}}\right)=\iint \chi_{a}(1) \chi_{b}(2)\left(1 / r_{12}\right) \chi_{a^{\prime}}(1) \chi_{b^{\prime}}(2) d v_{1} d v_{2} .
$$

Hybrid or ionic integrals :

$$
\left(\chi_{a} \chi_{a^{\prime}} \mid \chi_{a^{\prime \prime}} \chi_{b}\right)=\iint \chi_{a}(1) \chi_{a^{\prime}}(2)\left(1 / r_{12}\right) \chi_{a^{\prime \prime}}(1) \chi_{b}(2) d v_{1} d v_{2} .
$$

Exchange integrals:

$$
\left(\chi_{a} \chi_{b} \mid \chi_{b^{\prime}} \chi_{a^{\prime}}\right)=\iint \chi_{a}(1) \chi_{b}(2)\left(1 / r_{12}\right) \chi_{b^{\prime}}(1) \chi_{a^{\prime}}(2) d v_{1} d v_{2} .
$$

in which $r_{b}$ is the distance of the electron from nucleus $b$, (1) and (2) represent all the coordinates of electrons 1 and 2 respectively and $r_{12}$ is the distance between these electrons. The internuclear distance $R$ enters all the integrals as a parameter.

There are essentially only two methods of evaluating such integrals, one of which consists of using expansions of the reciprocals of the electron distances and the other expansions of the orbitals. The first method is based upon the pioneer work of Sugiura, ${ }^{3}$ Zener \& Guillemin, ${ }^{4}$ Bartlett, ${ }^{5}$ ROSEN ${ }^{6} \mathrm{JAMES}^{7}$ and others whilst the second is largely due to BARNETT \& Coulson $^{8}$ following a suggestion by CooliDGE. ${ }^{9}$ The choice of method for any particular problem is somewhat arbitrary and will probably be decided in the future by the availability of suitable tables.

The first method as used in the past has necessitated the computation of a large number of auxiliary integrals, defined as follows:

$$
\begin{gathered}
A_{n}(x)=\int_{1}^{\infty} e^{-x t} t^{n} d t ; \quad B_{n}(x)=\int_{-1}^{+1} e^{-x t} t^{n} d t ; \\
G_{\tau}{ }^{\nu}(n ; x)=\int_{-1}^{+1} e^{-x t} P_{\tau^{\nu}}(t) t^{m}\left(1-t^{2}\right)^{\nu / 2} d t,
\end{gathered}
$$

where $P_{\tau}{ }^{p}(t)$ is the associated Legendre function of the first kind ${ }^{10}$;

$$
f_{\tau}(m ; x)=\int_{1}^{\infty} Q_{\tau}(t) e^{-x t} t^{m} d t,
$$


where $Q_{\tau}{ }^{\nu}(t)$ is the associated Legendre function of the second kind $;^{10}$

$$
\begin{gathered}
F_{k, m, n}(x, y)=\int_{1}^{\infty} d t \int_{-1}^{+1} d t^{\prime}\left(t^{2}-1\right)^{k}\left(t+t^{\prime}\right)^{-(k+1)} t^{m} t^{\prime n} e^{-x t} e^{-y t^{\prime}} \\
J_{n}(x, y)=\int_{1}^{\infty} d t \int_{-1}^{+1} d t^{\prime}\left(t^{2}-1\right)\left(1-t^{\prime 2}\right)\left(t^{2}-t^{\prime 2}\right)\left(t+t^{\prime}\right)^{n} e^{-x t} e^{-y t^{\prime}} \\
K_{n}(x, y)=\int_{1}^{\infty} d t \int_{-1}^{+1} d t^{\prime}\left(t^{2}-1\right)\left(1-t^{\prime 2}\right)\left(1+t t^{\prime}\right)^{2}\left(t+t^{\prime}\right)^{n-2} e^{-x t} e^{-y t^{\prime}} ; \\
F_{m, n}(x, y)=\int_{1}^{\infty} d t \int_{-1}^{+1} d t^{\prime}\left(t+t^{\prime}\right)^{-m}\left(-t^{\prime}\right)^{n}\left(1-t^{\prime 2}\right)^{m-1} e^{-x t} e^{-y t^{\prime}} \\
S_{\tau}(m, n ; x)=\int_{1}^{\infty} d t Q_{\tau}(t) e^{-x t} t^{m} d t \int_{1}^{t} e^{-x t^{\prime}} t^{\prime n} d t^{\prime} ; \\
\sigma_{\tau}(m, n ; x)=\int_{1}^{\infty} d t Q_{\tau}(t) e^{-x t} t^{m} d t \int_{t}^{\infty} e^{-x t^{\prime}} t^{\prime n} d t^{\prime} ; \\
H_{\tau}(m, n ; x)=S_{\tau}(m, n ; x)+S_{\tau}(n, m ; x) \\
W_{\tau}^{\nu}(m, n ; x)=\int_{1}^{\infty} d t \int_{1}^{\infty} d t^{\prime} Q_{\tau}^{\nu}(t>) P_{\tau}^{\nu}(t<) e^{-x\left(t+t^{\prime}\right)} t^{m} t^{\prime n}\left(t^{\prime 2}-1\right)^{\nu / 2}
\end{gathered}
$$

All of these auxiliary integrals are expressable in terms of polynomials, exponentials, natural logarithms and the exponential integral $-\operatorname{Ei}(-x)=$ $\int_{x}^{\infty} t^{-1} e^{-t} d t$. Many of them are simply related to each other. There is a distressing lack of uniformity not only in the choice of auxiliary integrals computed but also in the notation.

The second method, which is particularly valuable in the case of integrals involving more than two centres, requires the computation of certain socalled $\zeta$-functions which are expressable ultimately in terms of Bessel functions of half-integral orders for which the argument is pure imaginary. ${ }^{11}$

Several approximations to the exchange and hybrid two-centre integrals and to multi-centred integrals have been suggested ${ }^{12}$ and their accuracy has recently been assessed by comparison with the exact values ${ }^{13}$.

This review concludes with three bibliographies;

$$
\begin{aligned}
& \text { A-Reduction of two-centre molecular integrals } \\
& \text { B-Tables of two-centre molecular integrals } \\
& \text { C-Multi-centre integrals. }
\end{aligned}
$$

A very large number of papers relevant to molecular integrals has been published and it is not possible (nor desirable) to describe all of them. Bibliography $\mathrm{A}$ is a somewhat arbitrary selection of papers chosen so that any integral that has been calculated to date may be found in at least one of them and chosen to include also those papers which the reviewer considers to be the most useful. Bibliography B describes in as much detail as space allows the tables of two-centre integrals and of auxiliary functions which 
have been published since the date of inception of $M T A C$ in 1943. Excluded from this bibliography is the multitude of published material which contains only a few isolated integrals and whose usefulness is effectively restricted to the problem concerned in the particular reference. Warning must be given that owing to the complexity of the work, the occurrence of errors is frequent, even the corrections being by no means exhaustive or completely reliable. The final bibliography $\mathrm{C}$ lists without description (apart from title) those papers concerned with multi-centre integrals. No systematic tabulation of multi-centre integrals has yet been published.

Information on tables published prior to 1943 may be obtained from the FMR Index ${ }^{14}$ and from the excellent bibliographies of MulligaN, ${ }^{15}$ RooTHAAN $^{16}$ and RÜDENBERG. ${ }^{17}$

Finally it seems worthwhile to mention that a discussion of future developments was reported in the proceedings of a conference on quantal methods in valence theory (QMVT). ${ }^{18}$

\section{Bibliography A}

\section{Reduction of two-centre molecular integrals.}

N. Rosen, "Calculations of interactions between atoms with $s$-electrons," Phys. Rev., v. 38, 1931, p. 255-276. Almost all types of molecular integrals involving identical $n s$ orbitals.

M. Kotani, A. Amemiya \& T. Simose, "Tables of integrals useful for the calculations of molecular energies I," Phys.-Math. Soc. Japan, Proc., v. 20, 1938, Extra no. 1, p. 1-70. Most of the integrals involving $n=2$ orbitals with a common screening parameter and a large number over $1 s$ and $n=2$ orbitals, the $n=2$ orbitals having a common screening parameter.

C. A. Coulson, "Two-centre integrals occurring in the theory of molecular structure," Camb. Phil. Soc., Proc., v. 38, 1941, p. 210-223. A wide selection of one electron integrals over $n s$ and $n p \sigma$ orbitals with no restriction on the screening parameters.

R. G. PARR \& B. L. CRAWFORD, JR., "On certain integrals useful in molecular orbital calculations," Jn. Chem. Phys., v. 16, 1948, p. 1049-1056. Most types of integrals over like $2 p \pi$ orbitals.

R. S. Mulliken, C. A. Rieke, D. Orloff \& H. Orloff, "Formulas and numerical tables for overlap integrals," Jn. Chem. Phys., v. 17, 1949, p. 1249-1267. Overlap integrals over all combinations of $n s, n p \sigma$, and $n p \pi$ AO's for $n=1,2,3,5$ with no restriction on the screening parameters.

H. J. Kopineck, "Austausch und andere Zweizentrenintegrale mit $2 s$ - und 2p-functionen," Zeits. Naturforschung, v. 5a, 1950, p. 420-431; "Zweizentrenintegrale mit $2 s$ - und $2 p$-functionen II. Ionenintegrale," Zeits. Naturforschung, v. 6a, 1951, p. 177-183. All the integrals involving only $n=2$ orbitals given in the second reference and some additional hybrid integrals.

G. Araki \& W. Watari, "Electronic states of $\mathrm{C}_{2}$-molecule III-Numerical values and reduction formulae of integrals," Prog. Theoretical Phys., v. 6, 1951, p. 961-979. The hybrid integrals over like $n=2$ orbitals not given in reference (A2). 
R. O. Brennan \& J. F. Mulligan, "Two-center heteronuclear hybrid coulomb-exchange integrals," Jn. Chem. Phys., v. 20, 1952, p. 1635-1644. All hybrid integrals involving $n=2$ orbitals with the restriction that orbitals on the same atom have a common screening parameter.

C. C. J. RoothaAn, "A study of two-center integrals useful in calculations on molecular structure I," Jn. Chem. Phys., v. 19, 1951, p. 1445-1458. All molecular integrals other than hybrid and exchange integrals over all combinations of orbitals with $n=1$ and 2 with no restriction on the screening parameters.

K. RÜDENBERG, "A study of two-center integrals useful in calculations on molecular structure II. Exchange integrals," Jn. Chem. Phys., v. 19, 1951, p. 1459-1477. Exchange integrals over all combinations of orbitals with $n=1,2,3$ with no restriction on the screening parameters.

M. Kotani, E. Ishiguro, K. Hijikata, T. Nakamura \& A. Amemiya, "Tables of integrals useful for the evaluation of molecular energies III," Phys. Soc. Japan, Jn., v. 8, 1953, p. 463-476. All the integrals over like $n=2$ orbitals not given in the earlier papers in this series.

These references are based upon the first method. The remaining references use the second method.

M. P. Barnett \& C. A. Coulson, "The evaluation of integrals occurring in the theory of molecular structure," Roy. Soc. Phil. Trans., v. 243A, 1951, p. 221-249; "The evaluation of unit molecular integrals," QMVT, 1951, p. 238-271. All molecular integrals.

\section{Bibliography B}

\section{Tables of two-centre molecular integrals.}

V. GRIFFING, "The size and vibration frequency of the excited benzene molecule," Jn. Chem. Phys., v. 15, 1947, p. 421-430. Most types of integrals over like $2 p \pi$ orbitals for $2 \zeta R=8 \cdot 87,8 \cdot 67(0 \cdot 1) 8 \cdot 17(0 \cdot 2) 7 \cdot 77$.

R. G. PARr \& B. L. CRAwford, JR., "Molecular orbital calculations of vibrational free constants I. Ethylene," Jn. Chem. Phys., v. 16, 1948, p. 526-532. Many integrals over like $2 p \pi$ orbitals for $2 \zeta R=6,8 \cdot 13,8 \cdot 37,10$. "On certain integrals useful in molecular orbital calculations," Jn. Chem. Phys., v. 16, 1948, p. 1049-1056. Many integrals over $2 p \pi$ orbitals for mainly $2 \zeta R=0,3$ (1) 10 .

R. S. Mulliken, C. A. Rieke, D. Orloff \& H. Orloff, "Formulas and numerical tables for overlap integrals," Jn. Chem. Phys., v. 17, 1949, p. 1249-1267. Overlap integrals over all combinations of $n=1,2,3,5$ orbitals usually for $\left(\zeta+\zeta^{\prime}\right) R=0$ (1) $16(2) 20,\left(\zeta-\zeta^{\prime}\right)\left(\zeta+\zeta^{\prime}\right)=-\cdot 6(\cdot 1) \cdot 6$. Also some linear combinations of these.

P. J. Wheatley \& J. W. Linnetr, "Molecular force fields Part XI-A wave mechanical treatment of the change with distortion of the interaction energy of carbon $2 p \pi$ orbitals," Farad. Soc., Trans., v. 45, 1949, p. 897-902. Most types of integrals over like $2 p \pi$ orbitals for $\zeta R=3.5(0 \cdot 5) 5$, 
J. O. Hirschfelder \& J. W. Linnett, "The energy of interaction between two hydrogen atoms," J. Chem. Phys., v. 18, 1950, p. 130-142. $S_{0}(m, n, x)$ for $x=.5(\cdot 5) 5(1) 8(2) 12,1.75 ; m=1$ (2) $5, n=0$ (2) 6 . $H(m, n, x)$ for $x=$ $0.5(0.5) 5$ (1) $8(2) 12,1 \cdot 75 ; m=0,2, n=0$ (2) 6 and $m=4, n=4,6$ and $m=6=n$. Many molecular integrals over $1 s$ and $n=2$ orbitals, all orbitals having same screening parameter for $\zeta R=0,1 \cdot 5,1 \cdot 75,2(\cdot 5) 5$ (1) 8 (2) 12.

H. J. Kopineck, "Austausch- und andere Zweizentrenintegrale mit 2sand $2 p$-functionen," Zeit. Naturforschung, v. 5a, 1950, p. 420-431. Most molecular integrals except hybrid integrals over $n=2$ orbitals with a common screening parameter $\zeta$ for $\zeta R=\cdot 5(\cdot 5) 7$ with a few omissions.

"Zweizentrenintegrale mit $2 s$ - und $2 p$-Funktionen II," Zeit. f. Naturfor-. schung, v. $6 a, 1951$, p. 177-183. Hybrid integrals over $n=2$ orbitals with a common screening parameter $\zeta$ for $\zeta R=\cdot 5(\cdot 5) 7$.

"Zweizentrenwechselwirkungsintegrale III. Integrale mit $2 p$ - und wasserstoffänhnlichen $2 s$-Funktionen," Zeit. Naturforschung, v. 7a, 1952, p. 785800. All types of integrals over $2 p$ and a linear combination of $1 s$ and $2 s$ orbitals with common screening parameter $\zeta$ for $\zeta R=1(\cdot 5) 7$.

G. Araki \& W. Watari, "Electronic states of $\mathrm{C}_{2}$-molecule," Prog. Theor. Phys., v. 6, 1951, p. 961-979. Many integrals over $n=2$ orbitals with a common screening parameter $\zeta$ for $\zeta R=1(\cdot 25) 5$.

M. P. Barnett \& C. A. Coulson, "The evaluation of integrals occurring in the theory of molecular structure," Roy. Soc. Phil. Trans., v. 243A, 1951, p. 221-249. Bessel functions of the first and second kinds of pure imaginary argument $I_{n+\frac{1}{3}}(x)$ and $K_{n+\frac{1}{3}}(x)$ for $n=-1(1) 4, x=0(\cdot 5) 10$ and $K_{n+\frac{1}{3}}(x)$ for $x=10$ (1) 25 .

A. A. Frost \& J. Braunstein, "Hydrogen molecule energy calculation by correlated molecular orbitals," Jn. Chem. Phys., v. 19, 1951, p. 1133-1138. $F_{010}(2 x, x)-F_{001}(2 x, x), F_{010}(x, 0)-F_{001}(x, 0)$ and $F_{010}(x,-x)-F_{001}$ $(x,-x)$ for $x=1(\cdot 5) 5$ (1) 7 and certain special integrals over like $1 s$ orbitals for $\zeta R=0(\cdot 5) 5$ (1) 7 .

K. F. Herzfeld \& V. Griffing, "Work on integrals at the Catholic University of America since 1949," QMVT, 1951, p. 202-204. Direct Coulomb attraction integrals over $2 p, 3 p$ and $4 p$ orbitals with a common screening parameter for $2 \zeta R=5,8,10(5) 20$.

E. Ishiguro, K. Hijikata, T. Arai \& M. Mizushima, Natural Science Reports, Ochanomizu University, v. 1, 1951, p. 22. Certain types of auxiliary integrals (cf. M. KotANI ${ }^{18} 1951$, p. 210).

[The reviewer has been unable to obtain this paper.]

J. F. MULLIGAN, "LCAO self-consistent field calculation of the ground state of carbon dioxide," Jn. Chem. Phys., v. 19, 1951, p. 347-362. Many integrals over $1 s(\zeta)$ and $n=2\left(\zeta^{\prime}\right)$ orbitals for $\zeta=5 \cdot 70,7 \cdot 70, \zeta^{\prime}=1 \cdot 625,2 \cdot 275$ and $R=2 \cdot 2$.

R. S. Mulliken, "Overlap and bonding power of $2 s, 2 p$-hybrid orbitals," Jn. Chem. Phys., v. 19, 1951, p. 900-912. Overlap integrals for $2 s, 2 p$-hybrid AO's of like atoms for $\zeta R=3.0(0 \cdot 2) 5(0.5) 7$. Some additional overlap integrals. 
E. Scrocco \& O. Salvetri, "Sul calcolo di certi integrali utili nel metods degli orbitale molecolari antisimmetrizzati," La Ricerca Scientifica, v. 21, 1951, p. 1629-1639. $\left(2 p \pi_{a} 2 p \pi_{a^{\prime}} \mid 2 p \pi_{a} 2 p \pi_{b^{\prime}}\right)$ for $\zeta R=0,4$ (2) 10, 14, 20; $\zeta^{\prime} / \zeta=\cdot 6(\cdot 2) 1.4$ with a few omissions.

"Studio di alcuni integrali che si presentano nei problemi di struttura molecolare," La Ricerca Scientifica, v. 22, 1952, p. 1766-1775. $\left(2 p \pi_{a} 2 p \pi_{b^{\prime}} \mid\right.$ $\left.2 p \pi_{a} 2 p \pi_{b^{\prime}}\right)$ for $\zeta R=4(2) 10,14,20 ; \zeta^{\prime} / \zeta=\cdot 6(\cdot 2) 1 \cdot 4$;

"Studio dell integrali di scambio e di ricerca di formule approximate per alcuni integrali che si incontrano nei problemi di struttura molecolare," La Ricerca Scientifica, v. 22, 1953, p. 98-107. $\left(2 p \pi_{a} 2 p \pi_{b}, \mid 2 p \pi_{b}, 2 p \pi_{a}\right)$ for $\zeta R=4(2) 10,14,20 ; \zeta^{\prime} / \zeta=0.6(0 \cdot 2) 1.4$

Y. AokI, "Numerical tables of overlap integrals for $3 s, 3 p$ hybrid A -0 ," Phys. Soc. Japan $J n$., v. 7, 1952, p. 451-457. Overlap integrals between $1 s$, $2 s, 2 p \sigma(\zeta)$ and hybrid $(3 s, 3 p \sigma)$ orbitals $\left(\zeta^{\prime}\right)$ for $\left(\zeta+\zeta^{\prime}\right) R=\cdot 0(1) 6(\cdot 4)$ 10 (1) 16 (2) 20 and $\left(\zeta^{\prime}-\zeta\right) /\left(\zeta^{\prime}+\zeta\right)=-\cdot 4(\cdot 1) 0 \cdot 3$; between hybrid $(3 s$, $3 p \sigma)$ orbitals for $\zeta^{\prime} R=0(5) \cdot 2(8) \cdot 5(10 \cdot 0)$.

R. O. Brennan \& J. F. Mulligan, "Two-center heteronuclear hybrid coulomb-exchange integrals," Jn. Chem. Phys., v. 20, 1952, p. 1635-1644. Hybrid integrals over $n=2$ orbitals such that orbitals on same atom have a common screening parameter for $\zeta=1.625, \zeta^{\prime}=2.275,(R=2.2)$.

I. Fischer, "The approximations of molecular theory tested on $\mathrm{LiH}$ and $\mathrm{BeH}^{+}, "$ Arkiv för Fysik, v. 5, 1952, p. 349-376. Some integrals over 1 s $(\zeta=1,2 \cdot 69)$ and $2 s(\zeta=\cdot 658)$ and $2 p(\zeta=\cdot 545)$ orbitals for $R=2 \cdot 64$, $3 \cdot 02,3 \cdot 40$ and $1 s(\zeta=1), 2 s(\zeta=\cdot 979), 2 p(\zeta=\cdot 871)$ for $R=2 \cdot 48$.

C. W. Jones, $A$ Short Table for the Bessel Functions $I_{n+\frac{1}{3}}(x), \frac{2}{\pi} K_{n+\frac{1}{3}}(x)$, 1952, University Press, Cambridge. $x^{-n-1} I_{n+\frac{1}{3}}(x)$ and $\frac{2}{\pi} x^{n+\xi} K_{n+\frac{1}{3}}(x)$ for $x=0(\cdot 1) 5, n=0(1) 10 ; e^{-x} I_{n+\frac{1}{3}}(x)$ and $\frac{2}{\pi} e^{x} K_{n+\frac{1}{3}}(x)$ for $x=5(\cdot 1) 10$, $n=0$ (1) 10 .

T. Murai \& G. Araki, "Calculation of heteronuclear molecular integrals," Prog. Theor. Phys., v. 8, 1952, p. 615-638. $J_{n}(x, y)$ and $K_{n}(x, y)$ for $n=-3$ (1) 2 and $x, y$ usually integers $\leq 10$ with some additional half integral values. $F_{m, n}(x, y)$ for $m=1, n=0$ (1) $9, x=1$ (1) 6 (1) $10, y=0$; also a large number of values of $F_{m, n}(x, y)$ for $m, n$ integers and $x, y$ usually integers in the ranges $0 \leq m \leq 4,0 \leq n \leq 9,2 \leq x \leq 10,0 \cdot 5 \leq y \leq 6 \cdot 5$. Integrals, mainly coulomb and hybrid over $n=2$ orbitals such that any two orbitals on the same atom have a common screening parameter.

I. G. Ross, "Calculations of the energy levels of acetylene by the method of antisymmetric molecular orbitals, including $\sigma-\pi$ interaction," Farad. Soc., Trans., v. 48, 1952, p. 973-991. Many integrals over $n=2$ orbitals with a common $\zeta$ for $\zeta R=3 \cdot 61$.

M. Kotani, E. Ishiguro, K. Hijikata, T. Nakamura \& A. Amemiya, "Tables of integrals useful for the calculations of molecular energies III," Phys. Soc. Japan, Jn., v. 8, 1953, p. 463-476. Extensive tables of molecular 
integrals over $n=2$ orbitals having a common screening parameter for $\zeta R=2(\cdot 25) 7$.

H. H. JAFFÉ \& G. O. DOAK, "On the stability of metallo-organic compounds," $J n$. Chem. Phys., v. 21, 1953, p. 196-200. Overlap integrals over $2 s, 5 s, 2 p \sigma$, $5 p \sigma$ and $3 d \sigma$ orbitals mainly for $\left(\zeta-\zeta^{\prime}\right) /\left(\zeta+\zeta^{\prime}\right)=-.75(\cdot 25) \cdot 75$, $\left(\zeta+\zeta^{\prime}\right)=0$ (1) $16,18,20$ (4) 32.

H. H. JAFFÉ, "Some overlap integrals involving XX orbitals," $J n$. Chem. Phys., v. 21, 1953, p. 258-263. Overlap integrals over $2 p \pi, 3 d \pi, 5 d \pi$ orbitals for $\left(\zeta-\zeta^{\prime}\right) /\left(\zeta+\zeta^{\prime}\right)=-.75(\cdot 25) \cdot 75,\left(\zeta+\zeta^{\prime}\right)=0$ (1) 16, 18, 20 (4) 32.

C. J. ThorNe, R. S. BARKer \& H. Eyring, "Tables of quantum mechanical integrals. I. Some two parameter integrals," Studies in A pplied Math. no. 10 (Univ. of Utah, Salt Lake City), 1953. Overlap and Coulomb repulsion integrals and others over $1 s$ orbitals for $R=1(\cdot 1) 1.5(\cdot 05) 2(\cdot 1) 5 ; \zeta=$ $.7(\cdot 05) 1(\cdot 02) 1.5$.

D. P. Craig, A. Maccoll, R. S. Nyholm, L. E. Orgel \& L. E. Sutton, "Chemical bonds involving $d$-orbitals. Part II," Chem. Soc. Jn., Jan. 1954, p. 354-357. Overlap integrals over some of the possible combinations of $n=3$ and $n=4$ orbitals usually for $\left(\zeta-\zeta^{\prime}\right) /\left(\zeta+\zeta^{\prime}\right)=-\cdot 5(\cdot 1) \cdot 5$, $\left(\zeta+\zeta^{\prime}\right) R=4(2) 12$ or $20 . B_{n}(x)$ for $x=0(\cdot 1) 1 \cdot 5, n=0$ (1) 8 .

R. S. BArker, H. Eyring, C. J. Thorne \& D. A. Baker, "Use of electron repulsion integral approximations in molecular quantum mechanics," $J n$. Chem. Phys., v. 22, 1954, p. 699-702. Hybrid and exchange integrals over 1 s orbitals for $R=1(\cdot 05) 3$ (1) $6,8,12$.

\section{Bibliography C \\ Multicentre integrals.}

A. London, "Theory of the ultraviolet absorption spectrum of diphenyl," Jn. Chem. Phys., v. 13, 1945, p. 396-428.

P. O. LöwDIn, A Theoretical Investigation into some Properties of Ionic Crystals, 1948, Almquist and Wicknell, Uppsala.

R. S. Mulliken, "Quelques Aspects de la Théorie des Orbitals Moléculaires," Jn. Chem. Phys., v. 46, 1949, p. 675-713.

C. A. Coulson \& M. P. BARnetr, "The evaluation of integrals occurring in the theory of molecular structure," Roy. Soc. Phil. Trans., v. 243A, 1951, p. 221-249; "The evaluation of unit molecular integrals," QMVT, 1951, p. 238-271.

J. F. MULLIGAN, "LCAO self-consistent field calculation of the ground state of carbon dioxide," Jn. Chem. Phys., v. 19, 1951, p. 347-362.

$\mathrm{K}$. RÜDENBERG, "On the three- and four-center integrals in molecular quantum mechanics," Jn. Chem. Phys., v. 19, 1951, p. 1433-1434.

R. TAYLOR, "Complete molecular orbital treatment of the system $\mathrm{H}_{4}$," Phys. Soc. Proc., v. 64A, 1951, p. 249-260.

I. FIsCher, "A theoretical study of the dipole moment of aniline," Arkiv. $f$. Fysik, v. 5, 1952, p. 377-389. 
S. O. LundQuist \& P. Löwdin, "On the calculation of certain integrals occurring in the theory of molecules, especially three-centre and four-centre integrals," Arkiv. f. Fysik, v. 3, 1952, p. 147-154.

P. O. Löwdin, "Approximate formulas for many center integrals in the theory of molecules and crystals," Jn. Chem. Phys., v. 21, 1953, p. 374-375.

R. S. Barker \& H. Eyring, "A study of three-center integrals useful in molecular quantum mechanics," Jn. Chem. Phys., v. 21, 1953, p. 912-917; "Evaluation of some electron repulsion integrals needed in molecular quantum mechanics. II. Use of the Neumann expansion in evaluation of two electron three-center repulsion integrals," Jn. Chem. Phys., v. 22, 1954, p. 114.

Queen's Univ. of Belfast

A. Dalgarno

Belfast, N. Ireland

* No notation has become standard yet.

1 J. SLATER, "Atomic shielding constants," Phys. Rev., v. 36, 1930, p. 57-64.

$2 \mathrm{~K}$. RÜDENBERG, "A study of two-center integrals useful in calculations on molecular structure II. Exchange integrals," Jn. Chem. Phys., v. 19, 1951, p. 1459-1477.

${ }^{3}$ Y. SugruRA, "Über die Eigenschaften des Wasserstoff Moleküls in Grundzustande," Zeit. Phys., v. 45,1927 , p. $484-492$.

${ }^{4}$ C. Zener \& V. Guillemin, "The $B$-state of the hydrogen molecule," Phys. Rev., v. 34, 1929 , p. $999-1009$.

'J. H. BartLetr, “Orbital valency,” Phys. Rev., v. 37, 1931, p. 507-531.

6 N. Rosen, "Calculation of interactions between atoms with s-electrons," Phys. Rev., v. 38, 1931, p. 255-276; "The normal state of the hydrogen molecule," Phys. Rev., v. 38, 1931, p. 2099-2114.

7 H. M. JAMES, "Wave-mechanical treatment of the $\mathrm{Li}_{2}$ molecule," Jn. Chem. Phys., v. 2, 1934, p. 794-810.

${ }^{8}$ M. P. BARNETT \& C. A. Coulson, "The evaluation of integrals occurring in the theory of molecular structure," Roy. Soc. London, Phil. Trans., v. 243A, 1951, p. 221-249.

9 A. S. Coolidge, "A quantum mechanics treatment of the water molecule," Phys. Rev., v. 42,1932 , p. $189-209$.

${ }_{10}$ E. W. HoBson, Spherical and Ellipsoidal Harmonics. Cambridge University Press, London, 1931.

${ }^{11} \mathrm{G}$. N. Watson, A Treatise on the Theory of Bessel Functions. Cambridge University Press, London, 1944.

12 (a) A. L. SKLAR, "The near ultraviolet absorption of substituted benzenes," $J_{n}$. Chem. Phys., v. 7, 1939, p. 984-993. (b) M. Goeppert Mayer \& K. J. McCallum, "Calculation of the absorption spectrum of Wurster's salts," Rev. Mod. Phys., v. 14, 1942, p. 248258. (c) R. S. Mulliken, "Quelques aspects de la theórie des orbitals moléculaires," Journ. Chim. Phys., v. 46, 1949, p. 497-542, 675-713. (d) J. F. Mulligan, "LCAO self-consistent field calculation of the ground state of carbon dioxide," Jn. Chem. Phys., v. 19, 1951, p. 347362. (e) S. L. AltmaNN, " $\pi-\sigma$ electronic states in molecules I. The Hückel approximation," Roy. Soc. London, Proc., v. 210A, 1952, p. 327-343. (f) E. Scrocco \& O. SAlverti, "Studio dell' integrale di scambio e di ricerca di formule approximate per alcuni integrali che si incontrano nei problemi di struttura molecolare," La Ricerca Scientifica, v. 23, 1953, p. 98-107. (g) C. R. MUELLER \& H. EYRING, "The overlap average and central field approximations," Jn. Chem. Phys., v. 19, 1951, p. 934-938. (h) H. PrEUSs, "Abschätzung für Zweizentrenintegrale," Zeit. f. Naturforschung, v. 8a, 1953, p. 270-272. (i) C. R. MUELLER, "Semilocalised orbitals IV. Relationship of $n$-center orbitals, two-center orbitals, and bond properties," Jn. Chem. Phys., v. 20, p. 1600-1604.

${ }_{13}$ (a) J. F. Mulligan-reference (12d). (b) R. O. Brennan \& J. F. Mulligan, "Twocenter heteronuclear coulomb-exchange integrals," Jn. Chem. Phys. v. 20, 1952, p. 16351644. (c) S. L. AltmanN-reference (12c). (d) E. Scrocco \& O. Salvetti-reference (12f). (e) C. R. Mueller \& H. Eyring-reference (12g). (f) H. Preuss-reference (12b). (g) C. R. Mueller-reference (12i). (h) R. S. Barker, H. Eyring, C. J. ThORNe \& D. A. BAKER, "Use of electron repulsion integral approximations in molecular quantum mechanics," Jn. Chem. Phys., v. 22, 1954, p. 699-702.

${ }^{14}$ A. Fletcher, J. C. P. Miller \& L. Rosenhead, An Index of Mathematical Tables, Scientific Computing Service, London 1946.

${ }^{15} \mathrm{~J}$. F. MULLIGAN-reference (12d). 
${ }^{16}$ C. C. J. RoothaAN, "A study of two-center integrals useful in calculations on molecular structure II," Jn. Chem. Phys., v. 19, 1951, p. 1445-1458.

${ }^{17} \mathrm{~K}$. RÜDENBERG-reference (2).

${ }_{18}$ Quantum-mechanical methods in valence theory, Sept. 1951, Nat. Acad. Sci. and Office of Naval Research, New York.

\section{Characters of the Symmetric Groups of Degree 15 and 16}

Introduction. The construction of the complete character table for the symmetric group of a given degree $n$ (i.e., the group formed by the permutations of $n$ quantities) is a problem of long standing. Theoretically the problem was solved many years ago by FroBENIUS, ${ }^{1}$ who first in troduced the concept of the character of a representation (thus meriting the title of founder of the theory of group representations). Frobenius' expression for the symmetric group characters as coefficients of a certain algebraic form is, however, unsuitable for practical calculation except for groups of quite low degree. Some years ago MURnaGHAN ${ }^{2}$ was able to derive a recursion scheme which permits one to calculate the characters for a given $n$ in terms of the (presumably known) characters of all the symmetric groups of lower degree. This scheme has been employed by various authors ${ }^{3}$ to construct character tables for all the symmetric groups of degree $n \leqslant 14$. The recursion scheme effectively reduces the problem to an exercise in bookkeeping, but, despite its simplicity, it suffers from two practical faults. First of all, the labor involved in computing the characters by hand becomes prohibitive for $n$ larger than, say, 14. Secondly, as mentioned above, for each $n$ the results for all lower $n$ must be used, so that errors may be expected to propagate. This, of course, is not a theoretical limitation, since in principle each table may be calculated, $a b$ initio, and any given table can be checked for consistency by using the fact that the characters themselves satisfy certain orthogonality relations. The impracticability of proceeding by hand computation is clear when it is remembered that the number of characters of the symmetric group of degree $n$ is equal to the square of the number of unrestricted partitions of $n$; for $n=15$, this number is 30,976 .

It should be evident that the practical difficulties mentioned in the preceding paragraph can in large measure be overcome by the use of electronic computers. In fact, it seemed to us that this very calculation would provide a very good test of the speed and flexibility of such a computer with regard to the handling of purely algebraic problems. Accordingly, we set up and carried out the calculation of the complete character tables of the symmetric groups of degree $n=15$ and 16 using the Los Alamos Electronic Computer (MANIAC). The method was checked by re-computing the tables ${ }^{4}$ for $n=10$ to $n=14$. The method of calculation is described in Sec. (III). The experience was quite encouraging, and suggests that it would be profitable to apply electronic computer techniques to a large class of quite complicated problems in algebra and group theory.

The Character Formulae. For convenience we give here a brief resume of the relevant properties of the symmetric group characters. For proofs and detailed discussion the reader is referred to the standard works of LitTLEWOOD $^{5}$ and MuRNAGHAN. ${ }^{6}$ 